\title{
The Fair Division of Hereditary Set Systems
}

\author{
Z. $\mathrm{Li}^{1}$ and A. Vetta ${ }^{2}$ \\ 1 Computer Science Department, École Normale Supérieure, Paris.zl@zli2.com ** \\ 2 Department of Mathematics and Statistics, and School of Computer Science, \\ McGill University. adrian.vetta@mcgill.ca
}

\begin{abstract}
We consider the fair division of indivisible items using the maximin shares measure. Recent work on the topic has focused on extending results beyond the class of additive valuation functions. In this spirit, we study the case where the items form an hereditary set system. We present a simple algorithm that allocates each agent a bundle of items whose value is at least 0.3667 times the maximin share of the agent. This improves upon the current best known guarantee of 0.2 due to Ghodsi et al. The analysis of the algorithm is almost tight; we present an instance where the algorithm provides a guarantee of at most 0.3738 . We also show that the algorithm can be implemented in polynomial time given a valuation oracle for each agent.
\end{abstract}

\section{Introduction}

Consider the problem of dividing up $m$ heterogenous goods amongst $n$ agents. How can this be achieved in an equitable manner? This is the classical problem of fair division in economics and political science [14. The issue that arises immediately is how to define "fairness". Two important concepts that have been widely studied are proportionality and envy-freeness. An allocation of the items to the agents is proportional if, for every agent, the value that the agent has for the grand bundle (all of the items) is at most a factor $n$ times greater than the value it has for the bundle it receives. The allocation is envy-free if the value an agent has for the bundle it receives is at least as great as the value it has for the bundle of any other agent; that is, no agent is willing to exchange its allocated bundle for the bundle of another agent ${ }^{3}$

Fair division has been extensively studied in the case of divisible items, typically in the general guise of cake-cutting [5|13]. Interestingly, for divisible items general equilibria can provide fair allocations in restricted settings. For example, assume the agents have linear valuation functions. If each agent is now given the same budget then equilibrium prices exist where all items are completely sold and each agent receives a most desired bundle; this concept of competitive equilibrium from equal incomes is due to Varian [15].

** The first author thanks McGill University for hosting them while conducting this research.

${ }^{3}$ Observe that if the agents have sub-additive valuation functions then envy-freeness implies proportionality. 
In practice, however, the fair division of indivisible items is more important than that of divisible items. This can be seen from the plethora of real-world examples, including course registration in universities, shift scheduling, draft assignment in sport, client assignment to sales-people, airport slot assignments, divorce settlements, and estate division [6]10. But, at first glance, it is not clear anything useful can be said regarding the fair division of indivisible goods. For instance, what is a fair way to allocate a single indivisible good between two agents? An important concept used in understanding the case of indivisible goods was introduced by Budish [6], namely, maximin shares. The basic protocol is familiar to every child when cake cutting: "I cut, you choose". More generally, for $n$ agents and $m$ indivisible goods, one agent partitions the items into $n$ bundles but that agent then gets the last choice of bundle. Intuitively, a risk averse agent seeks a partition that maximizes the value of its least desired bundle in the partition. The minimum value of a bundle in the optimal partition value is called the maximin share for the agent. Clearly, since the agents have different valuation functions, the optimal partitions and the corresponding maximin share values may differ for each agent. The first question that then arises is whether one can partition the items in such a way that every agent receives a bundle whose value is at least its maximin share. The answer is NO, even for additive valuation functions [10. This negative result leads to the question of whether or not approximate solutions exist. Specifically, is there a partition that gives every agent a bundle with value at least an $\alpha$-fraction of their maximin share? In groundbreaking work, for additive valuation functions, Kurokawa, Procaccia and Wang [10] showed the existence of a partition with $\alpha=\frac{2}{3}$; polynomial time algorithms with the same guarantee were subsequently given in [1] and [2]. A stronger guarantee of $\alpha=\frac{3}{4}$ was very recently obtained by Ghodsi et al. [8]. More general classes of valuation function have also been studied. Barman and Krishnamurthy 2] proved a bound of $\alpha=\frac{1}{10}$ for the class of submodular valuation functions. This was improved to $\frac{1}{3}$ by Ghodsi et al. [8, who also proved guarantees of $\frac{1}{5}$ for fractionally subadditive (XOS) valuations and $\Omega(1 / \log n)$ for subadditive valuations.

\subsection{Our Results.}

In this paper, we consider the fair division problem in an hereditary set system (or downward-closed set system). A set system $H=(J, \mathcal{F})$ consists of a collection $J$ of items and a family $\mathcal{F}$ of feasible (independent) subsets of $J$. The set system satisfies the hereditary property if:

$$
S \in \mathcal{F} \text { and } T \subset S \Longrightarrow T \in \mathcal{F}
$$

Hereditary set systems are ubiquitous in computer science and optimization. They arise naturally in the presence of packing or cost constraints, for example in scheduling problems and manufacturing processes. Furthermore, they are of fundamental theoretic importance; notable combinatorial and geometric objects that satisfy the hereditary property include matroids, simplicial complexes, and minor closed graph families such as networks embeddable on a surface. 
In an hereditary set system, each agent $i$ has a value $v_{i, j}$ for each item $j$ but these values are additive only on feasible sets in the set system. A formal description of this model is given in Section 2 along with a proposed algorithm for dividing up the items amongst the agents. Our main result, given in Section 3 , is that this algorithm provides a guarantee of at least 0.3667 for the maximin shares problem in a hereditary set system. This improves on the current best known bound of 0.2. In Section 4 we prove that our bound is almost tight by constructing an instance where the algorithm has a performance guarantee of at most 0.3738. Consequently, our lower and upper bounds for the performance guarantee of the algorithm are within an amount 0.007. The basic implementation of the algorithm runs in exponential time. So in Section 5 we show how to implement the procedure in polynomial time. Specifically, given a valuation oracle for each agent, the algorithm makes at most a polynomial in $m$ number of queries to the oracles and performs a polynomial amount of computation given the responses of the oracles.

\section{The Hereditary Maximin Share Problem}

In this section, we describe the maximin share problem on an hereditary set system. We present a fair division algorithm for the problem and provide a simple performance analysis of the procedure (which we improve upon in the next section).

\subsection{The Fair-Division Model.}

We have a set $I$ of $n$ agents and collection $J$ of $m$ items. The items belong to an hereditary set system $H=(J, \mathcal{F})$ and agents desire feasible (independent) sets in the set system. Specifically, each agent $i$ has an additive valuation function over independent sets. That is, for any $S \in \mathcal{F}$ we have $v_{i}(S)=\sum_{j \in S} v_{i, j}$. The value the agent has for a set $S \notin \mathcal{F}$ is simply the maximum value it has for any feasible subset of $S$; that is $v_{i}(S)=\max _{T \in \mathcal{F}: T \subset S} \sum_{j \in T} v_{i, j}$.

Our aim is to fairly divide up the items amongst the agents. We measure the fairness of a division with respect to the maximin share of each agent. To define this, let $\mathbb{P}$ be the set of all partitions of the items into $n$ sets. The value of the maximin share for a agent $i$ is then

$$
\operatorname{MMS}(i)=\max _{\mathcal{P} \in \mathbb{P}} \min _{P \in \mathcal{P}} v_{i}(P) .
$$

That is, the maximin share is a partition that maximizes the value of the least valuable bundle in the partition. A partition $\mathcal{P}_{i}=\left\{P_{i}^{1}, P_{i}^{2}, \ldots, P_{i}^{n}\right\} \in \mathbb{P}$ that attains this value is called a maximin partition for agent $i$ and the elements of $\mathcal{P}_{i}$ are called maximin parts. Observe that the maximin partition may be different for each agent.

Our objective is to find a partition of the items $\left\{S_{1}, S_{2}, \ldots, S_{\ell}\right\}$ where the bundle $S_{i}$ allocated to agent $i$ has value at least its maximin share. In general 
this is not possible, so instead we search for approximate solutions. Specifically, we desire the maximum fraction $\alpha>0$ and an allocation $\left\{S_{1}, S_{2}, \ldots, S_{\ell}\right\}$ such that $v_{i}\left(S_{i}\right) \geq \alpha \cdot \operatorname{MMS}(i)$, for every agent $i$. We call this the hereditary maximin share problem.

The hereditary maximin share problem has a constant factor approximation. This is because our valuation functions are fractionally subadditive (XOS). That is, the valuation function can be defined as the maximum over a collection of additive set functions. To show this, for each agent $i$, we define an additive function $a_{i}^{S}$ over the items for each independent set $S \in \mathcal{F}$. Specifically, let

$$
a_{i}^{S}(j)=\left\{\begin{array}{ll}
v_{i, j} & \text { if } j \in S \\
0 & \text { if } j \notin S
\end{array} .\right.
$$

It is then easy to verify, for any set $T$ (independent or not), that

$$
v_{i}(T)=\max _{S \in \mathcal{F}} \sum_{j \in T} a_{i}^{S}(j) .
$$

Thus $v_{i}$ is indeed fractionally subadditive. Using this fact, it follows from recent work of Ghodsi et al. $[8]$ that a performance guarantee of 0.2 is obtainable.

Theorem 1. [8] There is an algorithm for the fair division problem in hereditary set systems that allocates every agent a bundle with an approximation guarantee $\alpha=\frac{1}{5}$.

We remark that the valuation functions for hereditary set systems are not submodular functions 4 The aim of this paper is to improve upon the $\alpha=0.2$ performance guarantee.

\subsection{A Fair-Division Algorithm.}

To obtain a better performance guarantee we apply a simple and natural procedure. To begin, without loss of generality, we may assume that the maximin share of every agent is exactly 1 by normalizing. Even stronger, we may assume that, for every agent $i$, there exists a maximin partition such that the agent has value exactly 1 for each part in the partition. To see this formally, let $\mathcal{P}_{i}$ be a maximin partition for agent $i$. Now define a new valuation function $\hat{v}_{i}$ with the property that $\hat{v}_{i, j}=\frac{v_{i, j}}{v_{i}(P)}$ if item $j$ is in part $P \in \mathcal{P}_{i}$. Thus $\hat{v}_{i}(P)=1$, for each part $P \in \mathcal{P}_{i}$. It is then easy to verify that an allocation that provides a value $\alpha$ with respect to $\hat{v}_{i}$ is a factor $\alpha$ allocation with respect to the true valuation $v_{i}$.

\footnotetext{
${ }^{4}$ For example, consider an hereditary set system $H=(J, \mathcal{F})$ with three items $J=$ $\{a, b, c\}$ and let the maximal independent sets in $\mathcal{F}$ be $\{a\}$ and $\{b, c\}$. Suppose agent $i$ has item values $v_{i, a}=3, v_{i, b}=2$ and $v_{i, c}=2$. Thus $v_{i}(\{a, c\})=3$ and $v_{i}(\{a, b, c\})=4$. Consequently, the marginal value of adding item $c$ to the set $\{a, b\}$ is larger than the marginal value of adding item $c$ to the set $\{a\}$. Thus the valuation function is not submodular.
} 
We are now ready to present our fair-division algorithm which begins with the normalization above. This normalization is not required in our polynomial time algorithm; see Section 5 . Given a target value $\alpha$, we search for a minimum cardinality feasible sets of at least the targeted value for some agent. If such a set is found we allocate that set (bundle) to that agent and then recurse on the remaining items and agents. This method is formalized in Procedure 1.

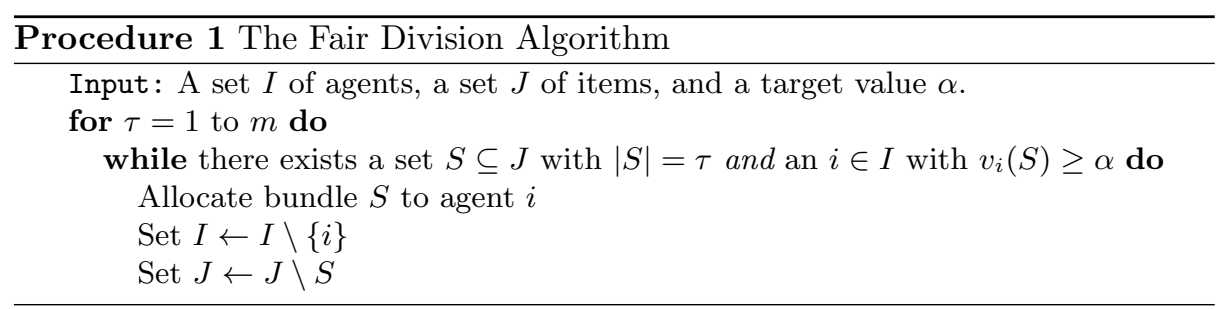

We use the following notation. Let $\left\{S_{1}, S_{2}, \ldots, S_{\ell}\right\}$ be the bundles assigned by the procedure in order; note that $\ell \leq n$. We view the procedure as working in phases. In Phase $\tau$ the procedure searches for bundles of cardinality $\tau$ that provide utility at least $\alpha$ for some agent; note that $\tau \leq m$. We denote by $\mathcal{A}_{\tau}$ the collection of all items allocated during Phase $\tau$.

\subsection{A Simple Analysis.}

To begin, let's present a very simple analysis that shows this algorithm gives a factor $\alpha=\frac{1}{3}$ guarantee. In Section 4 , we will give a more intricate and nearly tight analysis.

Theorem 2. The procedure allocates every agent a bundle of value at least $\alpha=$ $\frac{1}{3}$.

Proof. Clearly, if an agent is allocated a bundle by the procedure then it receives a bundle of value at least $\alpha$. So it suffices to show that the procedure allocates every agent a bundle if it is run with a target value $\alpha=\frac{1}{3}$. For a contradiction, suppose the procedure terminates after allocating bundles to $\ell<n$ agents. Let $i$ be an agent that is not allocated a bundle.

We may assume that $\mathcal{A}_{1}=\emptyset$. That is, $v_{i, j}<\alpha$ for every agent $i$ and every item $j$ and so no items are allocated in Phase 1. The argument is standard [10]: if a set of cardinality one is allocated to an agent then this item intersects at most one of the $n$ bundles in the maximin partition of any other agent. Thus $n-1$ of the bundles in the partition are untouched and each still have total value 1. Consequently, $n-1$ agents remain and they each have a partition of the items into $n-1$ bundles each with value 1 . Thus, we recurse on this smaller problem.

Therefore, we may assume the procedure only allocates items in Phases $\tau \geq 2$. Since the algorithm considers items in increasing size $\tau$, agents receive a minimal 
bundle with value the sum of value of its elements (by definition of valuation). Now take any set $S$ allocated to some agent $k$ in Phase $\tau$. It must be the case that $v_{i}(S)<\frac{\tau}{\tau-1} \cdot \alpha$. If not then there is a set $T \subset S$ with cardinality $\tau-1$ such that $v_{i}(T) \geq \frac{\tau-1}{\tau} \cdot \frac{\tau}{\tau-1} \cdot \alpha=\alpha$. But, by the hereditary property, the bundle $T$ is an independent set so should then have been allocated to agent $i$ in Phase $\tau-1$. Let bundle $S_{k}$ be the $k$ th bundle allocated, where $1 \leq k \leq \ell$. Let $U$ be the set of items unallocated by the procedure. Then the total value of unallocated items in some bundle of the maximin partition $\mathcal{P}_{i}$ of agent $i$ is at least

$$
\sum_{j \in U \cap \mathcal{P}_{i}} v_{i}(j) \geq n-\sum_{k=1}^{\ell} v_{i}\left(S_{k}\right) \geq n-\sum_{k=1}^{\ell} \frac{\left|S_{k}\right|}{\left|S_{k}\right|-1} \cdot \alpha \geq n-\sum_{k=1}^{\ell} 2 \cdot \alpha
$$

Here the final inequality arises as $\mathcal{A}_{1}=\emptyset$ and so $\left|S_{k}\right| \geq 2$ for every allocated bundle. Since $\alpha=\frac{1}{3}$, we obtain from (1) that

$$
\sum_{j \in U \cap \mathcal{P}_{i}} v_{i}(j) \geq n-\frac{2}{3} \ell>n-\frac{2}{3} n=\frac{n}{3}
$$

Because the maximin partition $\mathcal{P}_{i}$ contains exactly $n$ parts, there is a part that contains a set $S$ of unallocated items where $v_{i}(S) \geq \frac{n}{3} \cdot \frac{1}{n}=\frac{1}{3}$. By the hereditary property, this contradicts the fact that the procedure terminated without allocating agent $i$ a bundle. Thus every agent received a bundle of value at least $\frac{1}{3}$.

\section{An Improved Lower Bound}

In this section, we provide a much more detailed analysis of the fair division algorithm and prove it provides for an approximation guarantee of $\alpha=0.3667$. This analysis is almost tight; in Section 4 we present an example showing that the performance of the procedure is not better than $\alpha=0.3738$.

Theorem 3. The procedure allocates every agent a bundle of value at least $\alpha=$ $\frac{11}{30}$.

Before proving this theorem, let's give some intuition behind the analysis. The basic approach is the same as in Theorem 2 For an appropriately chosen target value $\alpha$ we run the procedure and assume for a contradiction that some agent $i$ was not allocated a bundle. We then consider the maximin shares partition $\mathcal{P}=\left\{P_{1}, P_{2}, \ldots, P_{n}\right\}$ for agent $i$ and show that some part $P \in \mathcal{P}$ contains items with total value at least $\alpha$ that are unallocated at the end of the procedure. By the hereditary property, this will contradict the fact the procedure terminated without allocating a bundle to agent $i$.

However, in order for this method to work for $\alpha=\frac{11}{30}$, we refine the analysis in four key ways. First, upon termination of the procedure, rather than considering the entire maximin shares partition $\mathcal{P}=\left\{P_{1}, P_{2}, \ldots, P_{n}\right\}$ for agent $i$, we focus on a restricted sub-partition $\hat{\mathcal{P}}$ of $\mathcal{P}$. To find $\hat{\mathcal{P}}$ we use combinatorial arguments 
on an auxiliary graph that is constructed with respect to the allocation decisions made in Phase 2 of the procedure. With this sub-partition $\hat{\mathcal{P}}$ more specialized accounting techniques can then be applied. We explain how to find the subpartition $\hat{\mathcal{P}}$ in Section 3.1 .

For the second improvement, note that upon termination we now wish to show that at least one of the parts $P \in \hat{\mathcal{P}}$ has unallocated items with total value $\alpha$. For this to not hold at the end of the procedure, we require that each part contains unallocated items of total value less than $\alpha$. This simple observation is useful in that once a part contains unallocated items of value less than $\alpha$, the removal of additional items from that part does not hurt us any more. In fact, the removal of additional items from such a part is actually beneficial to us in the analysis as such items do not reduce the value of parts that may still feasibly provide agent $i$ with a bundle of value $\alpha$. To quantify this, let

$$
v_{P}=1-\sum_{\tau \geq 1} \sum_{j \in P \cap \mathcal{A}_{\tau}} v_{i, j}
$$

be the total value of unallocated items in part $P \in \hat{\mathcal{P}}$ upon termination of the algorithm. We then denote by $s_{P}=\alpha-v_{P}$ the superfluity of part $P$. We may assume that $s_{P}>0$, otherwise the procedure would have allocated agent $i$ a bundle. Accounting for this superfluous damage will be the second key ingredient in the proof.

The third idea is to exploit any laxity the procedure provides before the start of the third phase. Essentially the laxity $l_{P}$ of a part $P \in \hat{\mathcal{P}}$ is a measure of how much better the unallocated value of the part is after Phase 2 than a "perceived" worst case. The fourth key idea is to amortize our accounting process. Rather than simply focus independently on items in each part of the partition $\hat{\mathcal{P}}$, we will also redistribute values within allocated bundles that cross multiple parts in the partition. The concepts of superfluity and laxity are explained in Section 3.2 along with a description of the amortization process. The proof of Theorem 3 is then given in Section 3.3

\subsection{Finding a Sub-Partition.}

Now assume the procedure terminates after $\ell<n$ iterations leaving at least one agent $i$ who does not receive a bundle. Let the maximin partition for agent $i$ be $\mathcal{P}=\left\{P_{1}, P_{2}, \ldots, P_{n}\right\}$. Let $J^{*} \subseteq J$ be the set of items allocated to agents during the procedure. We will show

$$
\max _{1 \leq k \leq n} v_{i}\left(P_{k} \backslash J^{*}\right) \geq \alpha
$$

This will contradict the fact that the procedure terminated without allocating a bundle to agent $i$. So let's prove that inequality (2) holds. As in the proof of Theorem 2, without loss of generality, we may assume no items were allocated in Phase 1 ; that is, $\mathcal{A}_{1}=\emptyset$. Next we construct an auxiliary graph $\mathcal{G}$ based upon the allocation decisions made in Phase 2. The graph contains $n$ vertices, one vertex 
for each part $P_{k}$ in the partition $\mathcal{P}$. The graph contains an edge connecting the two (possibly equal) parts containing the two items of the bundle, for each bundle allocated in Phase 2. Observe that a vertex in $\mathcal{G}$ may have degree greater than one since the part it represents may contain multiple items. This also implies that $\mathcal{G}$ may contain edges that are self-loops; this happens whenever a bundle allocated in Phase 2 consists of two items in the same part of the partition $\mathcal{P}$.

Let's further investigate the structure of $\mathcal{G}$. Let $X$ be a maximal set of vertices of $\mathcal{G}$ that induce at least $|X|$ edges. Note that such a set exists because $\emptyset$ is a feasible choice for $X$. On the other hand, it must be the case that $X \neq V(\mathcal{G})$. This follows as $\mathcal{G}$ contains exactly $n$ vertices but $\ell<n$ edges. But this, in turn, implies that $X$ induces exactly $|X|$ edges. If $X$ induced more than $|X|$ edges then we could add to it any other vertex in $V(\mathcal{G}) \backslash X$ and still maintain the desired property.

Now consider the subgraph $\mathcal{G} \backslash X$. This subgraph is a forest $F$. If it contained a cycle $C$ then $X \cup V(C)$ would contradict the maximality of $X$. Furthermore, there are no edges between $X$ and $\mathcal{G} \backslash X$; otherwise, the endpoint in $\mathcal{G} \backslash X$ of such an edge could have been added to $X$.

Let the forest $F$ contain $s$ components consisting of a single vertex - observe that, by the above argument, these vertices are also singleton components of $\mathcal{G}$. Let $F$ contain $c$ non-trivial components, that is, trees with at least one edge. Clearly, every non-trivial tree contains at least two leaves. Therefore, we may select a set $Y$ that consists of every vertex in non-trivial trees in $F$ except for exactly two leaves in each non-trivial tree. Finally, we set $Z=V(\mathcal{G}) \backslash(X \cup Y)$. An illustration of the auxiliary graph $\mathcal{G}$ and the sets $X, Y$ and $Z$ is shown in Figure 1.

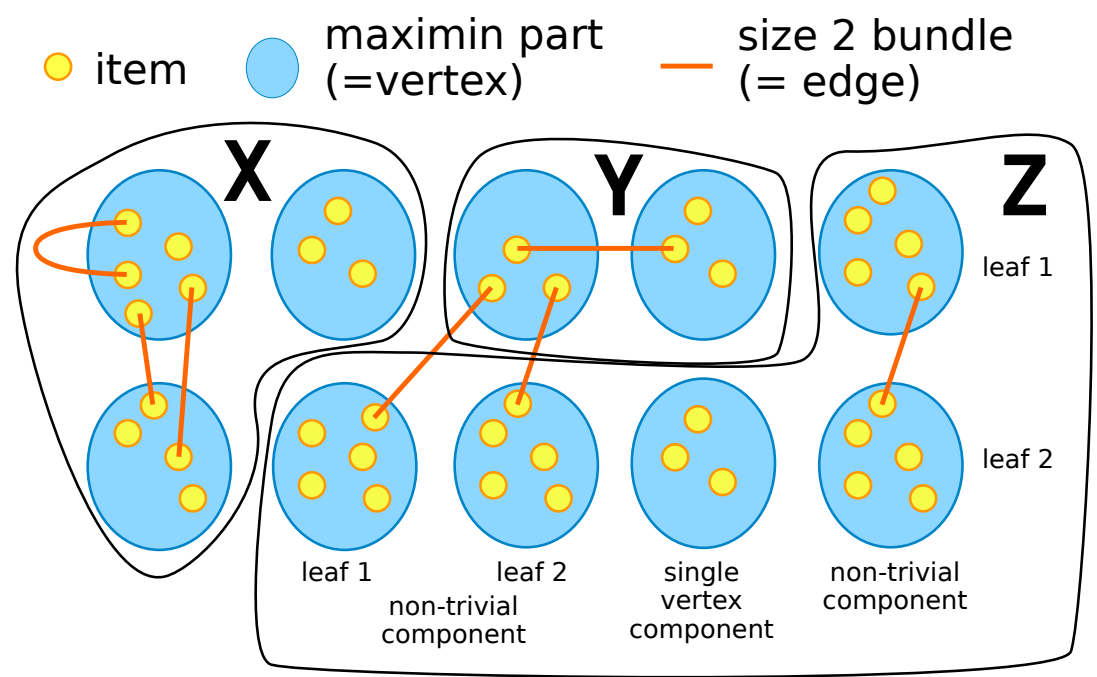

Fig. 1. The Auxiliary Graph. 
The sub-partition $\hat{\mathcal{P}}$ of $\mathcal{P}$ consisting of the vertices in $Z=V(\mathcal{G}) \backslash(X \cup Y)$ will be important to us. Let's now present a couple of combinatorial equalities that will be useful later. The first is a claim that follows trivially by definition of $\hat{\mathcal{P}}$. The second is a lemma quantifying how many agents are allocated bundles in Phase 2.

Claim. The number of parts in the sub-partition $\hat{\mathcal{P}}$ is $|Z|=2 c+s$.

Lemma 1. The number of agents allocated a bundle in Phase 2 is exactly $|X|+$ $|Y|+c$.

Proof. Observe that, by construction, the number of agents allocated a bundle in Phase 2 is exactly $|E(\mathcal{G})|$. So we must count the number of edges in the auxiliary graph $\mathcal{G}$. We have seen that $E(\mathcal{G})=E(X) \cup E(F)$. By the maximality of $X$, we have that $|E(X)|=|X|$. In addition, $|V(F)|=|Y|+2 c+s$. Thus, as $F$ consists of exactly $c+s$ trees, $|E(F)|=(|Y|+2 c+s)-(c+s)=|Y|+c$. Putting this together gives $|E(\mathcal{G})|=|X|+|Y|+c$, as desired.

We will focus our counting arguments on the sub-partition $\hat{\mathcal{P}}$ in order to obtain a contradiction to the fact that agent $i$ was not allocated a bundle. Specifically, we will show that at least one of the vertices in $Z$ contains unallocated items that together provide value at least $\alpha$ to agent $i$.

\subsection{Laxity, Superfluity and Amortization.}

Consider the allocated items in $\hat{\mathcal{P}}$. As $\mathcal{A}_{1}=\emptyset$, every item $j$ has $v_{i, j}<\alpha$. We now study the value (to agent $i$ ) of the items in $\hat{\mathcal{P}}$ allocated in Phase 2. To do this, recall that the vertices of $\hat{\mathcal{P}}=Z$ are of two types, vertices of degree 0 in $\mathcal{G}$ (specifically, singleton vertices in $F$ ) and vertices of degree 1 (that is, leaf vertices in $F$ ). Vertices of degree 0 contain no items that are allocated in Phase 2. Vertices of degree 1 in $\mathcal{G}$ contain exactly one item that is allocated in Phase 2. So given a part $P \in \hat{\mathcal{P}}$, we define the laxity of $P$ to be $l_{P}=\alpha$ if $P$ corresponds to a singleton vertex in $F$. Otherwise, if $P$ corresponds to a leaf vertex in $F$ we define $l_{P}=\left(1-v_{i, j^{*}(P)}\right)-(1-\alpha)=\alpha-v_{i, j^{*}(P)}$, where $j^{*}(P)$ is the unique item of $P$ that is allocated in Phase 2. Observe that $l_{P}>0$ since $v_{i, j}<\alpha$ for every item $j$ and in particular for $j^{*}(P)$. We can use the laxity to quantify the total value of items in $\hat{\mathcal{P}}$ allocated in Phase 2 .

Lemma 2. $\sum_{P \in \hat{\mathcal{P}}} \sum_{j \in P \cap \mathcal{A}_{2}} v_{i, j}=2 \alpha \cdot c-\sum_{P \in \hat{\mathcal{P}}} l_{P}$.

Proof. We have

$$
\sum_{P \in \hat{\mathcal{P}}} \sum_{j \in P \cap \mathcal{A}_{2}} v_{i, j}=\sum_{P \in \hat{\mathcal{P}}}\left(\alpha-l_{P}\right)=2 c \cdot \alpha-\sum_{P \in \hat{\mathcal{P}}} l_{P}
$$

Here the first equality holds since when $P$ corresponds to singleton, the associated term in the sum is $0=\alpha-\alpha=\alpha-l_{P}$, and when $P$ corresponds to a leaf, the associated term is $v_{i, j^{*}(P)}=\alpha-l_{P}$. The final equality follows as $\hat{\mathcal{P}}=Z$ contains exactly $2 \cdot c$ vertices of degree 1 . 
Next we want to bound the value (to agent $i$ ) of items allocated in Phases 3 and beyond. First, let's count the number of bundles allocated in Phases 3 and beyond.

Lemma 3. The number of agents allocated a bundle in Phases 3 and beyond is at most $c+s-1$.

Proof. By Lemma 1, the number of agents allocated a bundle in Phase 2 is exactly $|E(\mathcal{G})|=|X|+|Y|+c$. Consequently, as agent $i$ is not allocated a bundle in the procedure, the number of agents allocated bundles in Phases 3 and beyond is at most

$$
\begin{aligned}
(n-1)-|E(\mathcal{G})| & =(n-1)-(|X|+|Y|+c) \\
& =(|X|+|Y|+|\hat{\mathcal{P}}|-1)-(|X|+|Y|+c) \\
& =|\hat{\mathcal{P}}|-1-c \\
& =(2 c+s)-1-c \\
& =c+s-1
\end{aligned}
$$

Here the second equality arises because $n=|X|+|Y|+|Z|$ and $|Z|=|\hat{\mathcal{P}}|$; the fourth equality follows from Claim 3.1 .

Now let's bound the value of bundles allocated in Phases 3 and beyond. We will need two more definitions. First, recall, we defined the superfluity of a part $P$ as $s_{P}=\alpha-\left(1-\sum_{\tau \geq 1} \sum_{j \in P \cap \mathcal{A}_{\tau}} v_{i, j}\right)$. For the purpose of our analysis, we also define the excess $e_{P}$ of a part $P \in \hat{\mathcal{P}}$ to be the sum of its superfluity and its laxity. Therefore

$$
\begin{aligned}
e_{P} & =s_{P}+l_{P} \\
& =\left(\alpha-1+\sum_{\tau \geq 1} \sum_{j \in P \cap \mathcal{A}_{\tau}} v_{i, j}\right)+\left(\alpha-v_{i, j^{*}(P)}\right) \\
& =2 \alpha-1+\sum_{\tau \geq 3} \sum_{j \in P \cap \mathcal{A}_{\tau}} v_{i, j}
\end{aligned}
$$

The final equality holds because $\mathcal{A}_{1}=\emptyset$ and $P \cap \mathcal{A}_{2}$ is either $\left\{j^{*}(P)\right\}$ or $\emptyset$.

As discussed, to bound the value of bundles allocated in Phases 3 and beyond, we will amortize our accounting process. In these phases each allocated bundle has cardinality at least three. Take such a bundle, say $B=\left\{j_{1}, j_{2}, \ldots, j_{k}\right\}$ allocated to some agent $\tau$. The hereditary property states that $B \backslash\{j\}$ is feasible, for every item $j \in B$. Thus, because $B$ is a minimum cardinality feasible bundle of value at least $\alpha$ when it is allocated, it must be the case that $v_{i}(B \backslash\{j\})<\alpha$. Observe, this applies even for the least valuable item $\hat{j} \in B$ to agent $i$ in the bundle $B$. Furthermore, because $B$ is an independent set, we have that $(i) v_{i}(B \backslash\{\hat{j}\})=v_{i}(B)-v_{i, \hat{j}}$, and $(i i) v_{i, \hat{j}}$ is at most the average value of an item in $B$. Putting this all together gives

$$
v_{i}(B)<\alpha+v_{i, \hat{j}} \leq \alpha+\frac{1}{k} \cdot v_{i}(B)
$$


As $k \geq 3$, we obtain

$$
v_{i}(B)<\frac{k}{k-1} \cdot \alpha \leq \frac{3}{2} \cdot \alpha
$$

Hence, each bundle that is allocated in Phase 3 reduces the total value to agent $i$ of items in $\hat{\mathcal{P}}$ by at most $\frac{3}{2} \cdot \alpha$. For bundles of cardinality at least 4 (allocated in Phases 4 and beyond) this is at most $\frac{4}{3} \cdot \alpha$. This bound suffices for bundles of size at least 4 . We now bound bundles allocated in Phase 3 more carefully. To do so, we amortize the accounting process by defining for any item $j$ allocated in a bundle $B$ by the mechanism,

$$
a_{j}=\frac{v_{i}(B)}{|B|} .
$$

That is, $a_{j}$ is the average value of items in $B$. Since each allocated bundle is minimal and has cardinality 3 , we have, for any item $j$, that

$$
a_{j} \leq \frac{1}{3}\left(\alpha+v_{i, j}\right)
$$

We now further partition elements of $\mathcal{A}_{3}$ into two sets whose values we will bound independently in order to reduce the gaps in our accounting. To this end, let $U$ consist of those items in $\mathcal{A}_{3}$ that belong to a part $P \in \hat{\mathcal{P}}$ which contains no other elements of $\mathcal{A}_{3}$. That is, $j \in U$ if item $j$ is the only item of $\mathcal{A}_{3}$ in some part $P \in \hat{\mathcal{P}}$. Let $\bar{U}=\mathcal{A}_{3} \backslash U$; thus, $\bar{U}$ consists of those items in $\mathcal{A}_{3}$ that belong to a part $P \in \hat{\mathcal{P}}$ which contains a least two items of $\mathcal{A}_{3}$.

Claim. If $\alpha \leq \frac{11}{30}$ then for any part $P$ of $\hat{\mathcal{P}}$

$$
\sum_{j \in P \cap \bar{U}} a_{j}-e_{P} \leq \frac{1}{6} \cdot|P \cap \bar{U}|
$$

Proof. Take any part $P \in \hat{\mathcal{P}}$. If $P$ contains no elements of $\bar{U}$ then the claim is trivially true as, by definition, $e_{P} \geq 0$. Otherwise, by definition of $\bar{U}$, it must be the case that $P$ contains at least two elements of $\bar{U}$. Let $j_{1}$ be the most valuable and $j_{2}$ the second most valuable amongst items in $P \cap \bar{U}$. Assume $j_{1}$ was allocated in the bundle $B$ and $j_{2}$ was allocated in the bundle $B^{\prime}$, where possibly $B=B^{\prime}$. By the minimality of $B$, we have $v_{i}(B) \leq \alpha+v_{i, j_{1}}$. Since $j_{1} \in \bar{U} \subseteq \mathcal{A}_{3}$, we have that $|B|=3$ and so

$$
a_{j_{1}}=\frac{v_{i}(B)}{|B|} \leq \frac{\alpha+v_{i, j_{1}}}{|B|}=\frac{\alpha+v_{i, j_{1}}}{3}
$$

By the same argument, $a_{j_{2}} \leq \frac{\alpha+v_{i, j_{2}}}{3}$. Hence,

$$
\begin{aligned}
a_{j_{1}}+a_{j_{2}}-\frac{1}{3} \cdot e_{P} & \leq \frac{1}{3}\left(\alpha+v_{i, j_{1}}\right)+\frac{1}{3}\left(\alpha+v_{i, j_{2}}\right)-\frac{1}{3} e_{P} \\
& =\frac{2 \alpha}{3}+\frac{1}{3}\left(v_{i, j_{1}}+v_{i, j_{2}}-e_{P}\right) \\
& \leq \frac{2 \alpha}{3}+\frac{1}{3}\left(1-2 \alpha-\sum_{\tau \geq 3} \sum_{j \in P \backslash\left\{j_{1}, j_{2}\right\} \cap \mathcal{A}_{\tau}} v_{i, j}\right)
\end{aligned}
$$


Here, the final inequality follows by (3). Thus we have

$$
\begin{aligned}
a_{j_{1}}+a_{j_{2}}-\frac{1}{3} \cdot e_{P} & \leq \frac{1}{3}-\frac{1}{3} \cdot \sum_{\tau \geq 3} \sum_{j \in P \backslash\left\{j_{1}, j_{2}\right\} \cap \mathcal{A}_{\tau}} v_{i, j} \\
& \leq \frac{1}{3}-\frac{1}{3} \cdot \sum_{j \in P \backslash\left\{j_{1}, j_{2}\right\} \cap \mathcal{A}_{3}} v_{i, j} \\
& \leq \frac{1}{3}-\frac{1}{3} \cdot \sum_{j \in P \backslash\left\{j_{1}, j_{2}\right\} \cap \bar{U}} v_{i, j}
\end{aligned}
$$

Hence

$$
\begin{aligned}
\sum_{j \in P \cap \bar{U}} a_{j}-e_{P} & =\left(a_{j_{1}}+a_{j_{2}}-\frac{1}{3} \cdot e_{P}\right)+\sum_{j \in P \backslash\left\{j_{1}, j_{2}\right\} \cap \bar{U}} a_{j}-\frac{2}{3} \cdot e_{P} \\
& \leq\left(\frac{1}{3}-\frac{1}{3} \cdot \sum_{j \in P \backslash\left\{j_{1}, j_{2}\right\} \cap \bar{U}} v_{i, j}\right)+\sum_{j \in P \backslash\left\{j_{1}, j_{2}\right\} \cap \bar{U}} a_{j}-\frac{2}{3} \cdot e_{P} \\
& \leq\left(\frac{1}{3}-\frac{1}{3} \cdot \sum_{j \in P \backslash\left\{j_{1}, j_{2}\right\} \cap \bar{U}} v_{i, j}\right)+\frac{1}{3} \cdot \sum_{j \in P \backslash\left\{j_{1}, j_{2}\right\} \cap \bar{U}}\left(\alpha+v_{i, j}\right)-\frac{2}{3} \cdot e_{P}
\end{aligned}
$$

The above inequalities follow from (8) and (5), respectively. Consequently,

$$
\begin{aligned}
\sum_{j \in P \cap \bar{U}} a_{j}-e_{P} & \leq \frac{1}{3}+\frac{1}{3} \cdot \sum_{j \in P \backslash\left\{j_{1}, j_{2}\right\} \cap \bar{U}} \alpha-\frac{2}{3} \cdot e_{P} \\
& \leq \frac{1}{3}+\frac{1}{3} \cdot \sum_{j \in P \backslash\left\{j_{1}, j_{2}\right\} \cap \bar{U}} \alpha \\
& =\frac{1}{6}+\frac{1}{6}+\frac{\alpha}{3} \cdot(|P \cap \bar{U}|-2) \\
& \leq \frac{1}{6} \cdot|P \cap \bar{U}|
\end{aligned}
$$

Here the final inequality follows as $\alpha=\frac{11}{30} \leq \frac{1}{2}$. This completes the proof of the claim.

Applying Claim 3.2 to every part $P \in \hat{\mathcal{P}}$, we obtain that

$$
\sum_{j \in \bar{U}} a_{j} \leq \frac{1}{6} \cdot|\bar{U}|+\sum_{P \in \hat{\mathcal{P}}} e_{P}
$$

Moreover, because $a_{j} \leq \frac{1}{2} \cdot \alpha$ for all $j \in U \subseteq \mathcal{A}_{3}$, we also have

$$
\sum_{j \in U} a_{j} \leq \frac{1}{2} \cdot \alpha \cdot|U|
$$




\subsection{Proof of the Improved Bound.}

We are now ready to prove the stated performance guarantee of the procedure.

Theorem 3, The procedure allocates every agent a bundle of value at least $\alpha=$ $\frac{11}{30}$.

Proof. Let $\beta_{3}$ be the number of bundles of size 3 allocated in Phase 3 and let $\beta_{4^{+}}$be number of bundles of size at least 4 allocated in Phase 4 and beyond. Applying inequality (4) for $k \geq 4$, we have

$$
\sum_{\tau \geq 4} \sum_{j \in \mathcal{A}_{\tau}} v_{i, j} \leq \frac{4}{3} \cdot \alpha \cdot \beta_{4^{+}}
$$

This gives

$$
\begin{aligned}
\sum_{\tau \geq 3} \sum_{j \in \mathcal{A}_{\tau}} v_{i, j} & =\sum_{j \in \mathcal{A}_{3}} v_{i, j}+\sum_{\tau \geq 4} \sum_{j \in \mathcal{A}_{\tau}} v_{i, j} \\
& \leq \sum_{j \in \mathcal{A}_{3}} v_{i, j}+\frac{4 \alpha}{3} \cdot \beta_{4^{+}} \\
& =\sum_{j \in \mathcal{A}_{3}} a_{j}+\frac{4 \alpha}{3} \cdot \beta_{4^{+}} \\
& =\sum_{j \in U} a_{j}+\sum_{j \in \bar{U}} a_{j}+\frac{4 \alpha}{3} \cdot \beta_{4^{+}}
\end{aligned}
$$

where the last equality follows by definition of $U$ and $\bar{U}$. Applying 11 and 10 then produces:

$$
\begin{aligned}
\sum_{\tau \geq 3} \sum_{j \in \mathcal{A}_{\tau}} v_{i, j} & \leq \frac{\alpha}{2} \cdot|U|+\left(\frac{1}{6} \cdot|\bar{U}|+\sum_{P \in \hat{\mathcal{P}}} e_{P}\right)+\frac{4 \alpha}{3} \cdot \beta_{4^{+}} \\
& =\frac{\alpha}{2} \cdot|U|+\frac{1}{6} \cdot\left(\left|\mathcal{A}_{3}\right|-|U|\right)+\frac{4 \alpha}{3} \cdot \beta_{4^{+}}+\sum_{P \in \hat{\mathcal{P}}} e_{P} \\
& =\left(\frac{\alpha}{2}-\frac{1}{6}\right) \cdot|U|+\frac{1}{6} \cdot\left|\mathcal{A}_{3}\right|+\frac{4 \alpha}{3} \cdot \beta_{4^{+}}+\sum_{P \in \hat{\mathcal{P}}} e_{P} \\
& \leq\left(\frac{\alpha}{2}-\frac{1}{6}\right) \cdot|U|+\frac{1}{2} \cdot \beta_{3}+\frac{4 \alpha}{3} \cdot \beta_{4^{+}}+\sum_{P \in \hat{\mathcal{P}}} e_{P} \\
& \leq\left(\frac{\alpha}{2}-\frac{1}{6}\right) \cdot|U|+\frac{1}{2} \cdot\left(\beta_{3}+\beta_{4^{+}}\right)+\sum_{P \in \hat{\mathcal{P}}} e_{P}
\end{aligned}
$$

Here the second inequality is due to the fact that $\left|\mathcal{A}_{3}\right|=3 \beta_{3}$; the final inequality follows as our target value is $\alpha=\frac{11}{30} \leq \frac{3}{8}$. 
Now, by definition, there is at most one element of $U$ for each of the $2 c+s$ maximin parts in $\hat{\mathcal{P}}$. So $|U| \leq 2 c+s$. Furthermore, by Lemma 3 , we have $\beta_{3}+\beta_{4^{+}} \leq c+s-1$. Therefore, it follows that

$$
\sum_{\tau \geq 3} \sum_{j \in \mathcal{A}_{\tau}} v_{i, j} \leq\left(\frac{\alpha}{2}-\frac{1}{6}\right)(2 c+s)+\frac{1}{2}(c+s)+\sum_{P \in \hat{\mathcal{P}}} e_{P}
$$

We are now ready to complete the proof. The total value of non-superflous, allocated items in $\hat{\mathcal{P}}$ at the end of the procedure is then at most

$$
\begin{aligned}
\sum_{\tau \geq 1} \sum_{j \in \mathcal{A}_{\tau}} v_{i, j}-\sum_{P \in \hat{\mathcal{P}}} s_{P} & =\sum_{j \in \mathcal{A}_{1}} v_{i, j}+\sum_{j \in \mathcal{A}_{2}} v_{i, j}+\sum_{\tau \geq 3} \sum_{j \in \mathcal{A}_{\tau}} v_{i, j}-\sum_{P \in \hat{\mathcal{P}}} s_{P} \\
& =0+\left(2 \alpha c-\sum_{P \in \hat{\mathcal{P}}} l_{P}\right)+\sum_{\tau \geq 3} \sum_{j \in \mathcal{A}_{\tau}} v_{i, j}-\sum_{P \in \hat{\mathcal{P}}} s_{P} \\
& \leq 2 \alpha c-\sum_{P \in \hat{\mathcal{P}}} l_{P}+\left(\left(\frac{\alpha}{2}-\frac{1}{6}\right)(2 c+s)+\frac{1}{2}(c+s)+\sum_{P \in \hat{\mathcal{P}}} e_{P}\right)-\sum_{P \in \hat{\mathcal{P}}} s_{P}
\end{aligned}
$$

Here the second equality follows from the fact that $\mathcal{A}_{1}=\emptyset$ and by Lemma 2 . The inequality follows by (13). Simplifying now gives

$$
\begin{aligned}
\sum_{\tau \geq 1} \sum_{j \in \mathcal{A}_{\tau}} v_{i, j}-\sum_{P \in \hat{\mathcal{P}}} s_{P} & \leq 2 \alpha c+\left(\frac{\alpha}{2}-\frac{1}{6}\right)(2 c+s)+\frac{1}{2}(c+s) \\
& =2 c \cdot\left(\alpha+\frac{1}{2} \alpha-\frac{1}{6}+\frac{1}{4}\right)+s \cdot\left(\frac{1}{2} \alpha-\frac{1}{6}+\frac{1}{2}\right) \\
& =2 c \cdot\left(\frac{1}{12}+\frac{3}{2} \alpha\right)+s \cdot\left(\frac{1}{3}+\frac{1}{2} \alpha\right)
\end{aligned}
$$

By Claim 3.1, at the start of the procedure the total value of items to agent $i$ in the sub-partition $\hat{\mathcal{P}}$ is at least $(2 c+s) \cdot 1$. Thus upon termination, the total value of unallocated items (modulo superfluity) is at least

$$
\begin{aligned}
2 c+s-\left(\sum_{\tau \geq 1} \sum_{j \in \mathcal{A}_{\tau}} v_{i, j}-\sum_{P \in \hat{\mathcal{P}}} s_{P}\right) & \geq(2 c+s)-\left(2 c \cdot\left(\frac{1}{12}+\frac{3}{2} \alpha\right)+s \cdot\left(\frac{1}{3}+\frac{1}{2} \alpha\right)\right) \\
& =2 c \cdot\left(\frac{11}{12}-\frac{3}{2} \alpha\right)+s \cdot\left(\frac{2}{3}-\frac{1}{2} \alpha\right) \\
& \geq(2 c+s) \cdot\left(\frac{11}{12}-\frac{3}{2} \alpha\right)
\end{aligned}
$$

Here the final inequality holds because $\frac{11}{12}-\frac{3}{2} \alpha \leq \frac{2}{3}-\frac{1}{2} \alpha$ for $\alpha=\frac{11}{30} \geq \frac{1}{4}$. Hence the average remaining value in each part of $\hat{\mathcal{P}}$ at the end of the procedure is at least $\frac{11}{12}-\frac{3}{2} \alpha$. This is at least $\alpha$ for $\alpha \leq \frac{11}{30}$. Thus agent $i$ must receive a bundle of value at least $\alpha=\frac{11}{30}=0.3667$. 


\section{An Upper Bound}

We now show that the analysis in Section 3 is tight to within an additive amount of 0.007. Specifically, we present an example that shows the procedure cannot guarantee a performance guarantee better than $\frac{40}{107}=0.3738$.

Theorem 4. The performance guarantee of the procedure is at most $\frac{40}{107}=$ 0.3738 .

Proof. Set $\alpha^{\prime}=\frac{40}{107}$. We will construct an hereditary set system with the property that the procedure will fail to allocate every agent a bundle if we select a target value of $\alpha=\alpha^{\prime}+\varepsilon$, for any arbitrarily small constant $\varepsilon>0$.

The set system contains six classes of items, denoted $\{A, B, C, D, E, F\}$. The number of identical items in each class are shown in the second row of Table 1. We assume the number $n$ of agents is a multiple of $2 \cdot 3 \cdot 5 \cdot 11=330$. Moreover these agents are identical. The value each agent has for a single item of each class is shown in the third row of the table. Finally, the feasible (independent) sets are defined by a capacity constraint for each class of items, as shown in third row of Table 1. Specifically, a set $S$ is feasible if it contains at most 2 items of class $A$, at most 1 item of class $B$, at most 2 items of class $C$, at most 5 items of class $D$, at most 11 items of class $E$, and at most 40 items of class $F$.

\begin{tabular}{|c||c|c|c|c|c|c|}
\hline Class & $\mathrm{A}$ & $\mathrm{B}$ & $\mathrm{C}$ & $\mathrm{D}$ & $\mathrm{E}$ & $\mathrm{F}$ \\
\hline Quantity & $n$ & $\frac{1}{3} n$ & $\frac{2}{3} n$ & $\frac{2}{3} n$ & $\frac{1}{3} n$ & $40 n$ \\
\hline Value & $\alpha^{\prime}=\frac{40}{107}$ & $\frac{13}{4} \alpha^{\prime}-1=\frac{23}{107}$ & $1-\frac{9}{4} \alpha^{\prime}=\frac{17}{107}$ & $\frac{1}{4} \alpha^{\prime}=\frac{10}{107}$ & $2-\frac{21}{4} \alpha^{\prime}=\frac{4}{107}$ & $\frac{1}{40} \alpha^{\prime}=\frac{1}{107}$ \\
\hline Capacity & 2 & 1 & 2 & 5 & 11 & 40 \\
\hline
\end{tabular}

Table 1. The Hereditary Set System

As feasibility is defined only by a capacity constraint on each item, it immediately follows that this set system satisfies the hereditary property. (In fact, this set system is a partition matroid which prompts an interesting open problem that we discuss in the conclusion.)

We claim that there is a partition $\mathcal{P}=\left\{P_{1}, P_{2}, \ldots, P_{n}\right\}$ where each part has value exactly 1 . Specifically, this maximin partition consists of parts of two types. There are $\frac{2}{3} n$ parts of Type I: each part contains exactly one item from all the classes $A, C, D$ and forty items from class $F$. There are $\frac{1}{3} n$ parts of Type II: each part contains exactly one item from all the classes $A, B, E$ and forty items from class $F$. Notice that in total we use $n$ items of class $A, \frac{2}{3} n$ items from classes $C$ and $D, \frac{1}{3} n$ items from classes $B$ and $E$, and $40 n$ items from classes $F$. Hence, this is a valid partition. Moreover, each of these parts is an independent 
set as the capacity constraint for each class is clearly satisfied. These parts all have value exactly one because

$$
v(A, C, D, 40 F)=\frac{40}{107}+\frac{17}{107}+\frac{10}{107}+40 \cdot \frac{1}{107}=\frac{107}{107}=1
$$

and

$$
v(A, B, E, 40 F)=\frac{40}{107}+\frac{23}{107}+\frac{4}{107}+40 \cdot \frac{1}{107}=\frac{107}{107}=1 .
$$

Now let's examine what the procedure does when faced with this instance. It may allocate bundles as follows:

- Phase 2: $\frac{1}{2} n$ agents each receive 2 items of class $A$.

- Phase 3: $\frac{1}{3} n$ agents each receive 1 item of class $B$ and 2 items of class $C$.

- Phase 5: $\frac{2}{15} n$ agents each receive 5 items of class $D$.

- Phase 11: $\frac{1}{33} n$ agents each receive 11 items of class $E$.

To verify that this is a valid output of the procedure, it is necessary to check that all the bundles above were of minimum cardinality at the time they were allocated. By construction, there are no items with value at least $\alpha$. Therefore, no bundles are allocated in Phase 1. Thus the bundles consisting of two items of class $A$ are indeed minimum cardinality feasible bundles when they are allocated in Phase 2. After Phase 2, there are no items of class $A$ remaining. Thus, because of the capacity one constraint on items of class $B$, it is easy to see that every feasible bundle of value at least $\alpha$ must contain at least three items. In particular, taking one item from class $B$ and one item of class $C$ gives total value $\frac{40}{107}=\alpha-\varepsilon$. Thus, in Phase 3 the bundles consisting of 1 item of class $B$ and 2 items of class $C$ are of minimum cardinality at the time of allocation. After $\frac{1}{3} n$ such bundles have been allocated then are now no items of classes $B$ or $C$; hence only items of the classes $D, E$ and $F$ remain. As the maximum value of any item in these classes is $\frac{10}{107}$, it is now not possible to obtain any feasible bundles of cardinality 4 with value at least $\alpha$. Thus the bundles allocated in Phase 5 are indeed of minimum cardinality; this exhausts the supply of items of class $D$. Now the maximum value of a remaining item is only $\frac{4}{107}$ so there are no feasible bundles of cardinality at most 10. Consequently, the bundles allocated in Phase 11 are also all of minimum cardinality; these bundles also exhaust the supply of items of class $E$.

Notice, at this point, we have allocated bundles to $\left(\frac{1}{2}+\frac{1}{3}+\frac{2}{15}+\frac{1}{33}\right) \cdot n=$ $\frac{329}{330} \cdot n$ of the agents. Therefore, we must now verify why no further bundles are allocated in Phase 12 and beyond. After Phase 11 all the items from classes $A, B, C, D$ and $E$ have been allocated. Thus, only the items of class $F$ are left. But the capacity constraint on class $F$ implies that no agent can obtain value $\alpha$ using only items in $F$. Therefore the procedure terminates at this point with $\frac{1}{330} n$ of the agents receiving no bundle.

\section{A Polynomial Time Implementation}

Procedure 1 is not a polynomial time algorithm. However, it can be modified to give a polynomial time implementation given access to a valuation oracle for 
each agent. To do this there are two main problems. First, the use of a phase $\tau$ to search for bundles of cardinality $\tau$ is clearly exponential time if the procedure ends up searching for bundles of large cardinality. Second, the procedure, requires the maximin partition or, more specifically, the maximin share value for each agent.

Here we detail how to overcome both these problems using a polynomial amount of computation and a polynomial number of valuation queries. Specifically, we present an implementation of Procedure 1 that runs in time polynomial in the number of items $m$. (We may assume the number of items $m$ is at least the number of agents, otherwise each maximin share value is 0 .) Note that the hereditary set system that is part of the input may be very large compared to the number of items 5 Therefore, henceforth, we make the standard assumption that the valuation functions are given via a valuation oracle. Specifically, for each agent $i$, we possess an oracle which returns $v_{i}(S)$ when given a subset $S$ of the items as a query. Thus, our aim is to prove that the modified algorithm makes only a polynomial number of calls to the valuation oracles and preforms only a polynomial amount of additional computations.

\subsection{Overview of the Polytime Algorithm.}

Before diving into the formal proof, we discuss the intuition behind the algorithmic modifications. Recall there are two main problems we must overcome. First, if $\tau$ is large then searching over bundles of cardinality $\tau$ in Phase $\tau$ is exponential time. This problem is quite easy to deal with. We implement Phases 1,2 and 3 as before but then we group together Phases 4 through to $m$. We do this by finding a minimal cardinality bundle $S$ with value at least $\alpha$ for some agent. That is, $v_{i}(S) \geq \alpha$ for some agent $i$, and $v_{\ell}(S \backslash\{j\})<\alpha$ for any agent $\ell$ and any item $j \in S$. We then allocate bundle $S$ to a remaining agent who values it the most. Finding such a minimal set can be done in polynomial time. Moreover, this grouping of phases does not affect the performance guarantee as the proof of Theorem 3 already implicitly groups together Phases $\{4,5, \ldots, m\}$ in the analysis.

The second problem concerns the fact that the procedure uses the maximin share value $\operatorname{MMS}(i)$ for each agent $i$. Specifically, this is used to scale the valuation functions so that the total value of every part in the maximin share partition is exactly one. Note that Procedure 1 does not require the maximin share partitions of the agents. Moreover, the analysis in the proof of Theorem 3 requires only that value of each part is at least one (or at least MMS $(i)$ before scaling) rather than exactly one. So if we could calculate the MMS $(i)$ then we would be done. Unfortunately, we cannot efficiently calculate $\operatorname{MMS}(i)$ so we must estimate it. For

\footnotetext{
${ }^{5}$ Indeed, for $m$ items, there are doubly exponentially many possible hereditary set systems. For example, let $\mathcal{J}_{m / 2}$ be the set of bundles of cardinality exactly $m / 2$. Then any subset $\mathcal{J}$ of $\mathcal{J}_{m / 2}$ induces a distinct hereditary set system. There are $2^{\left(\begin{array}{c}m \\ m / 2\end{array}\right)}$ such set systems and so we would need at least $\left(\begin{array}{c}m \\ m / 2\end{array}\right)$ bits to represent such an hereditary set system.
} 
additive valuation functions there is a PTAS for calculating the maximin share value [16] but this does not extend to hereditary set systems; so an alternate approach is required. The basic approach we take is as follows. Suppose we had guesses $\mu_{i}$ for $\operatorname{MMS}(i)$ for each $i$ and that all these guesses are known to be overestimates. Then imagine running Procedure 1 with valuations $v_{i}^{\prime}=v_{i} / \mu_{i}$. Next, suppose we get lucky and each agent is allocated a bundle. Now, because the $\mu_{i}$ are overestimates, each agent $i$ receives a bundle of value (with respect to $v_{i}$ ) of at least $\alpha \cdot \mu_{i} \geq \alpha \cdot \operatorname{MMS}(i)$.

But what do we do if some agent $i$ is not allocated a bundle? In this case we decrease $\mu_{i}$ to obtain new estimates and repeat the procedure again - in fact, we can decrease $\mu_{i}$ for all agents who receive no bundle. We decrease $\mu_{i}$ by multiplying it by a constant factor $1-\delta$, for some very small constant $\delta$. After doing so $\mu_{i}$ does not underestimate $\operatorname{MMS}(i)$ by worse than a $1-\delta$ factor. The key to the proof is then to show that the algorithm must allocate agent $i$ a bundle if we underestimate $\operatorname{MMS}(i)$. Hence the procedure will never further decrease $\mu_{i}$ once it drops below MMS $(i)$. It then remains to show that we only need to repeat the procedure at most a polynomial number of times; this will follow by obtaining suitable upper and lower bounds on each MMS $(i)$.

\subsection{A Formal Description of the Polytime Algorithm.}

We now formalize the intuitive description give above and prove the resultant procedure runs in time polynomial in the number of items $m$. Formally, our polynomial time algorithm is described in Procedure 2.

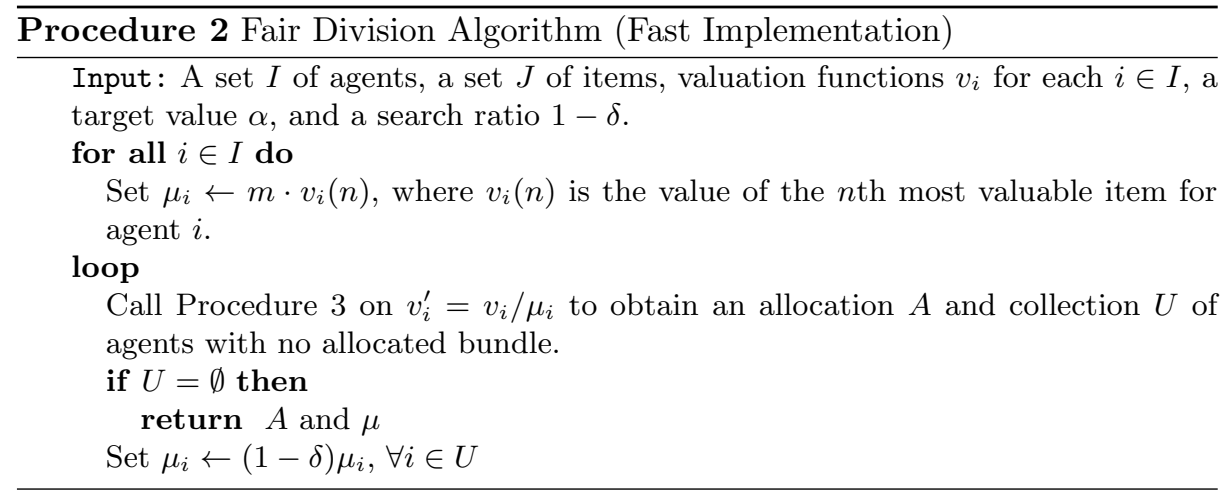

The algorithm calls a sub-procedure, Procedure 3, which groups together Phases 4 to $n$. This grouping, in turn, relies upon Procedure 4 which given a target value $\alpha$, finds a minimal cardinality bundle of items of value at least $\alpha$ and returns this bundle together with the agent who values it the most.

We then obtain the following theorem whose proof is the same as Theorem 3 . 


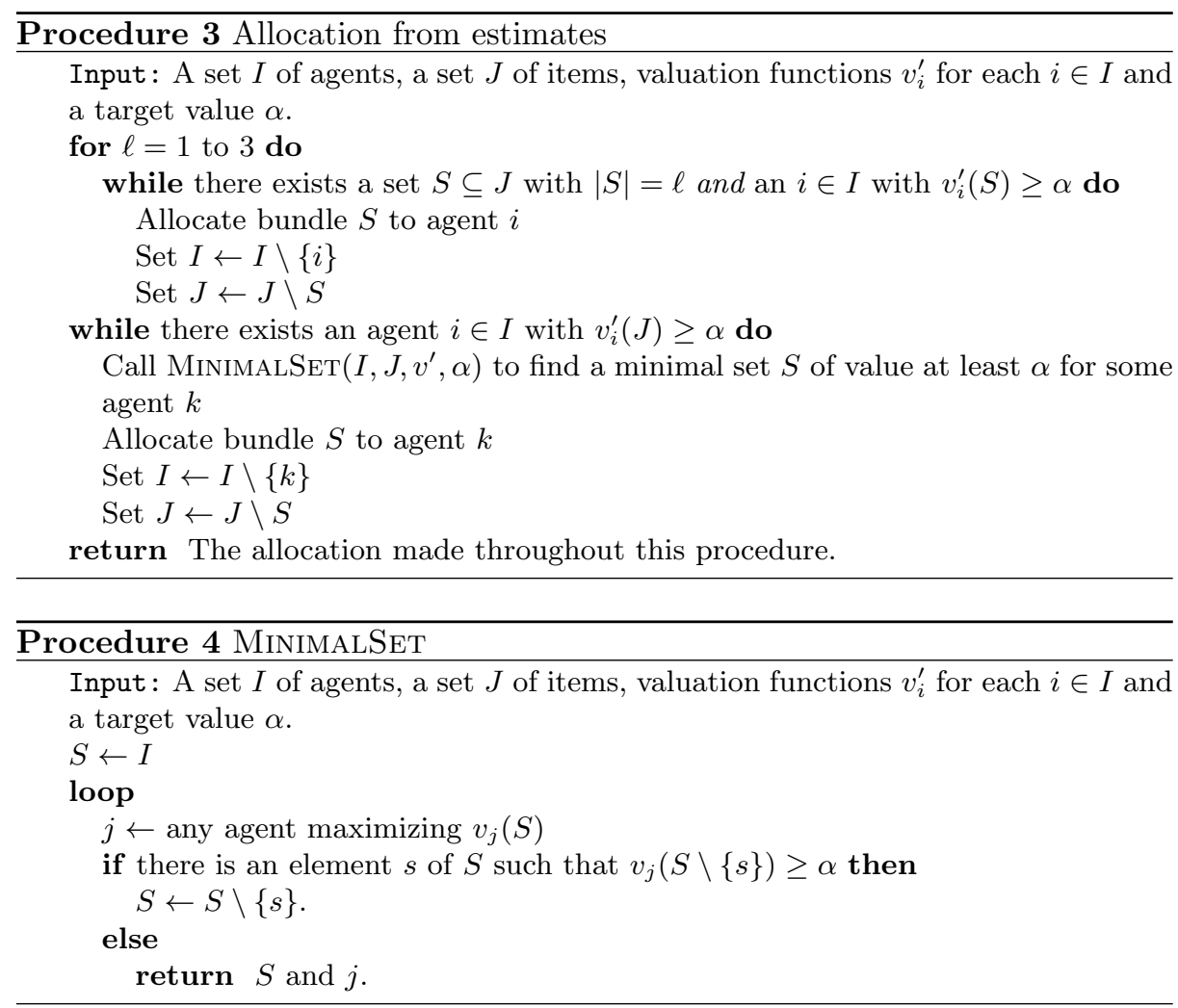

Theorem 5. Let $\mu_{1}, \ldots, \mu_{n}$ be positive constants. If we run Procedure 3, with valuations $v_{i}^{\prime}=v_{i} / \mu_{i}$ and target $\alpha=\frac{11}{30}$, then every agent $i$ with $\mu_{i} \leq \operatorname{MMS}(i)$ is allocated a bundle $S_{i}$ of value $v_{i}\left(S_{i}\right) \geq \alpha \cdot \mu_{i}$.

The contrapositive of Theorem 5 is that any agent who does not receive a bundle has $\mu_{i}>\operatorname{MMS}(i)$. Using this we may prove the main theorem of this section.

Theorem 6. Procedure 2 produces an allocation where every agent $i$ receives a bundle of value at least $(1-\delta) \cdot \alpha \cdot \operatorname{MMS}(i)$.

Proof. We prove this by induction on the number of iterations of the loop in Procedure 2. In fact, we prove the stronger statement that $\mu_{i} \geq(1-\delta) \cdot \operatorname{MMS}(i)$ for every agent $i$ throughout the procedure. Thus, in the last call to Procedure 3. each agent receives a bundle (as $U=\emptyset$ ) of value $\alpha \cdot \mu_{i} \geq(1-\delta) \cdot \alpha \cdot \operatorname{MMS}(i)$. For the base case, before the first iteration, we have $v_{i}(J) \geq \operatorname{MMS}(i)>(1-\delta) \cdot \operatorname{MMS}(i)$ for each agent $i$. Next suppose $\mu_{i} \geq(1-\delta) \operatorname{MMS}(i)$ holds in some iteration $\ell$; we now show it still holds in the iteration $\ell+1$. There are two cases. First, if $i \notin U$ then $\mu_{i}$ is unchanged in this iteration and so $\mu_{i} \geq(1-\delta) \cdot \alpha \cdot \operatorname{MMS}(i)$ is true in the next iteration. Second, if $i \in U$ then, by the contrapositive of Theorem 5 . 
we have that $\mu_{i}>\operatorname{MMS}(i)$. Then since $\mu_{i}$ is multiplied by a factor $(1-\delta)$, in the next iteration we have $\mu_{i} \geq(1-\delta) \cdot \operatorname{MMS}(i)$ as required.

We remark that we can run Procedure 2 with $n$ identical copies of an agent to obtain a partition giving a $(1-\delta) \cdot \frac{11}{30}$ approximation to the maximin share value of that agent.

Theorem 7. There is a $(1-\delta) \cdot \frac{11}{30}$-approximation algorithm for the maximin share problem on hereditary set families for any $\delta>0$.

\subsection{The Running Time.}

Let's verify that this algorithm needs at most a polynomial number of valuation queries.

Procedure 4 requires at most $m$ iterations as we remove at least one item each iteration. In each iteration, it takes $|S| \leq m$ queries to find whether there is an item removable from $S$ and we may attempt to do this for all $n$ agents. Thus, Procedure 4 runs in polynomial time.

Procedure 3 first searches all bundles of size at most three using $O\left(n \cdot m^{3}\right)$ queries (by asking each agent for their value of each bundle of size at most three) and then runs at most $n$ iterations of Procedure 4.

The initialization step of Procedure 2, for each of the $m$ agents, $n$ queries and $O(m)$ time is used to find the $n$th most valuable item. Each iteration of Procedure 2 updates at most $m$ entries of $\mu$. It remains to upper bound the number of iterations of Procedure 2 required. To compute this, we need upper and lower bounds on $\operatorname{MMS}(i)$.

Claim. Let $v_{i}(n)$ be the value of the $n$th most valuable item for agent $i$. Then $m \cdot v_{i}(n) \geq \operatorname{MMS}(i) \geq v_{i}(n)$.

Proof. For the lower bound consider any partition $\mathcal{P}=\left\{P_{1}, P_{2}, \ldots, P_{n}\right\}$ where $P_{k}$ contains the $k$ th most valuable item for agent $i$, for any $1 \leq k \leq n$. Then clearly $v_{i}\left(P_{k}\right) \geq v_{i}(k) \geq v_{i}(n)$. Thus, $\operatorname{MMS}(i) \geq v_{i}(n)$.

Next consider the upper bound. Take the maximin partition $\mathcal{P}$ for agent $i$. Then there is some part $P_{k}$ that does not contain any of the $n-1$ most valuable items for agent $i$. Since all the other items have value at most $v_{i}(n)$ we have that $v_{i}\left(P_{k}\right) \leq m \cdot v_{i}(n)$. Thus, $\operatorname{MMS}(i) \leq m \cdot v_{i}(n)$.

The ratio between these upper and lower bounds for MMS $(i)$ in Claim 5.3 is $m$. As our estimates for $\operatorname{MMS}(i)$ decrease in $(1-\delta)$ factors we have that the number of iterations required is at most $n \cdot \log _{1 /(1-\delta)} m=O(n \cdot \ln m)$.

Putting everything together we see that the total number of demand queries and the total computation time is polynomial in $m$. 


\section{Conclusion}

We have presented a fair division algorithm for hereditary set systems which provides each agent with at least an $\frac{11}{30}$ fraction of its maximin share value. Several open problems remain. The first is to close the 0.007 gap between the lower and upper bounds given for the performance of the procedure given in this paper. The second is to design a new procedure with a better performance guarantee. Of course, this may be easier to do for sub-classes of hereditary set systems. One very important sub-class is that of matroids. A matroid is a hereditary set system that also satisfies the augmentation property: given two independent sets $S$ and $T$ where $|S|>|T|$, there exists an element $s \in S$ such that $T \cup\{s\}$ is independent. Because the almost-tight example presented in Section 4 is a (partition) matroid, the procedure presented in this paper does not have a better performance guarantee for matroids. So a third open problem would be to design a fair division algorithm that exploits the augmentation property to produce improved performance guarantees for matroids 6

Acknowledgements. The authors thank Jugal Garg, Vasilis Gkatzelis and Richard Santiago for interesting discussions on fair division. We are also grateful to Dominik Peters for useful pointers to relevant literature. Finally, we thank the anonymous reviewers for helpful suggestions.

\section{References}

1. G. Amanatidis, E. Markakis, A. Nikzad and A. Saberi, "Approximation algorithms for computing maximin share allocations", ACM Transactions on Algorithms, 13(4), Article \#52, 2018.

2. S. Barman and S. Krishna Murthy, "Approximation algorithms for maximin fair division", Proceedings of the 18th Conference on Economics and Computation (EC), pp647-664, 2017.

3. S. Bouveret, K. Cechlarova, E. Elkind, A. Igarashi and D. Peters, "Fair division of a graph", Proceedings of the 26th International Joint Conference on Artificial Intelligence (IJCAI), pp135-141, 2017.

4. S. Bouveret and J. Lang, "Characterizing conflicts in fair division of indivisible goods using a scale of criteria", Proceedings of 13th Conference on Autonomous Agents and Multi-Agent Systems (AAMAS), pp1321-1328, 2014.

5. S. Brahms and D. King, Fair Division: From Cake Cutting to Dispute Resolution, Cambridge University Press, 1996.

6. E. Budish, "The combinatorial assignment problem: approximate competitive equilibrium from equal incomes", Journals of Political Economy, 119(6), pp1061-1103, 2011.

7. I. Caragiannis, D. Kurokawa, H. Moulin, A. Procaccia, N. Shah and J. Wang, "The unreasonable fairness of maximum nash welfare", Proceedings of 16th Conference on Economics and Computation (EC), pp305-322, 2016.

${ }^{6}$ We remark that 9] considers the maximin share problem in matroids but under a different model where, in addition to each agent being assigned a feasible set, the union of all these bundles must also be a feasible set. 
8. M. Ghodsi, M. HajiAghayi, M. Seddighin, S. Seddighin and H. Yami, "Fair allocation of indivisible goods: Improvement and generalization", Proceedings of the 19th Conference on Economics and Computation (EC), pp539-556, 2018.

9. L. Gourvès and J. Monnot, "On maximin share allocations in matroids", Theoretical Computer Science, 754, pp50-64, 2019.

10. D. Kurokawa, A. Procaccia and J. Wang, "Fair enough: guaranteeing approximate maximin shares", Journal of the ACM, 65(2), Article \#8, 2018.

11. H. Moulin, "Uniform externalities: two axioms for fair allocation", Journal of Public Economics, 43(3), pp305-326, 1990.

12. H. Moulin, Fair Division and Collective Welfare, MIT Press, 2003.

13. J. Robertson and W. Webb, Cake Cutting Algorithms: Be Fair if you can, A K Peters, 1998.

14. H. Steinhaus, "The problem of fair division", Econometrica, 16, pp101-104, 1948.

15. H. Varian, "Equity, envy and efficiency", Journal of Economic Theory, 9, pp63-91, 1974.

16. G. Woeginger, "A polynomial time approximation scheme for maximizing the minimum machine completion time", Operations Research Letters, 20, pp149-154, 1997. 